BMJ Open

Diabetes

Research

\& Care

\section{Discovery of plasma biomarkers for predicting the severity of coronary artery atherosclerosis by quantitative proteomics}

To cite: Ku EJ, Cho K-C, Lim C, et al. Discovery of plasma biomarkers for predicting the severity of coronary artery atherosclerosis by quantitative proteomics. BMJ Open Diab Res Care 2020;8:e001152. doi:10.1136/ bmjdrc-2019-001152

- Additional material is published online only. To view, please visit the journal online (http://dx.doi.org/10.1136/ bmjdrc-2019-001152).

EJK and K-CC contributed equally.

Received 24 December 2019 Revised 4 March 2020 Accepted 24 March 2020
Check for updates

(C) Author(s) (or their employer(s)) 2020. Re-use permitted under CC BY-NC. No commercial re-use. See rights and permissions. Published by BMJ.

For numbered affiliations see end of article.

Correspondence to Professor Sung Hee Choi; shchoimd@gmail.com and Professor Kwang Pyo Kim; kimkp@khu.ac.kr

\section{ABSTRACT}

Introduction Cardiovascular disease (CVD) in patients with diabetes is the leading cause of death. Finding early biomarkers for detecting asymptomatic patients with CVD can improve survival. Recently, plasma proteomicstargeted selected reaction monitoring/multiple reaction monitoring analyses (MRM) - has emerged as highly specific and sensitive tools compared with classic ELISA methods. The objective was to identify differentially regulated proteins according to the severity of the coronary artery atherosclerosis.

Research design and methods A discovery cohort, a verification cohort and a validation cohort consisted of 18,53 , and 228 subjects, respectively. The grade of coronary artery stenosis was defined as a percentage of luminal stenosis of the major coronary arteries. Participants were divided into six groups, depending on the presence of diabetes and the grade of coronary artery stenosis. Two mass spectrometric approaches were employed: (1) conventional shotgun liquid chromatography tandem mass spectrometry for a discovery and (2) quantitative MRM for verification and validation. An analysis of the covariance was used to examine the biomarkers' predictivity beyond conventional cardiovascular risks.

Results A total of 1349 different proteins were identified from a discovery cohort. We selected 52 proteins based on the tandem mass tag quantitative analysis then summarized as follows: chemokine (C-X-C motif) ligand 7 (CXCL7), apolipoprotein C-II (APOC2), human lipopolysaccharide-binding protein (LBP) and dedicator of cytokinesis 2 (DOCK2) in diabetes; CXCL7, AP0C2, LBP, complement 4A (C4A), vitamin D-binding protein (VTDB) and laminin $\beta 1$ subunit in non-diabetes. Analysis of covariance showed that APOC2, DOCK2 CXCL7 and VTDB were upregulated and C4A was downregulated in patients with diabetes showing severe coronary artery stenosis. LBP and VTDB were downregulated in patients without diabetes, showing severe coronary artery stenosis.

Conclusion We identified significant associations between circulating APOC2, C4A, CXCL7, DOCK2, LBP and VTDB levels and the degree of coronary artery stenosis using the MRM technique.

\section{Significance of this study}

What is already known about this subject?

- Recent quantitative proteomics technologies have enabled the identification of potential biomarkers of various chronic inflammatory diseases.

- Among quantitative proteomics, selected reaction monitoring (SRM)/multiple reaction monitoring (MRM) analysis is a highly sensitive and reproducible method for the targeted quantitation of protein/ peptide.

- Cardiovascular disease is major cause of mortality in patients with diabetes, but there are rare distinguished biomarkers, especially from early stage of atherosclerosis.

What are the new findings?

- We categorized the groups according to the severity of coronary artery disease (from normal to severe) and performed SRM/MRM analysis using each blood sample.

- After validation with the well-known cardiovascular risk factors together, we provide the characteristics of protein/peptide in patients with and without diabetes. Upregulated apolipoprotein C-II, dedicator of cytokinesis 2 and chemokine ligand seven and downregulated complement $4 \mathrm{~A}$ were significantly associated with severity of coronary artery stenosis in patients with diabetes.

How might these results change the focus of research or clinical practice?

- Our findings suggests that monitoring of circulating protein/peptides using SRM/MRM analysis may be potentially effective for finding proper targets for the prevention or treatment strategy to protect against atherosclerosis in advance.

\section{BACKGROUND}

Cardiovascular disease (CVD) associated with atherosclerosis is the leading cause of mortality worldwide. ${ }^{1}$ Up to $50 \%$ of patients 
with diabetes have CVD, including coronary artery sclerosis, stroke, peripheral arterial diseases, and other heart diseases. ${ }^{2}$ Notably, the vascular lesion in diabetes is very diffuse and worse than that observed in other patients; therefore, an early diagnosis of atherosclerotic lesions is especially important in patients with diabetes. ${ }^{3}$ In these patients, coronary arterial disease (CAD) is commonly asymptomatic in the early, and even in the later stages, and diagnosis can be delayed because of atypical symptoms. In recent years, several clinical risk factors for $\mathrm{CAD}$ in diabetes have been identified, for example, smoking, hyperglycemia, hypercholesterolemia, sheer stress on vessels, and inflammatory cytokines related to chronic inflammation. ${ }^{3}$ Because of the heterogeneity and complexity of both diabetes mellitus and atherosclerosis, the implementation of screening tools for the early diagnosis of subclinical to overt atherosclerosis is highly desirable for enhancing the survival rate.

Recently, a molecular approach was used to identify biomarkers for the diagnosis of several degenerative diseases. ${ }^{4}$ However, to date, few have been applied in clinical practice because of difficulties in developing applicable and replicable biomarkers and their immunoassays. Recent advances in the field of proteomics incorporating mass spectrometry (MS) are highlighted, as they have provided protein biomarkers that are closely related to the pathophysiology of their respective conditions. MS-based proteomics experiments detect all proteins within a sample based on their mass-to-charge ratio. Beyond the traditional MS technique, the selected reaction monitoring (SRM)/multiple reaction monitoring (MRM) approach used in tandem MS is highly specific and sensitive because it allows the fine detection of peptides or protein fragments of interest. ${ }^{5}$

In the present study, we evaluated blood samples from control individuals and patients with CAD to identify novel biomarkers of atherosclerosis progression based on the severity of coronary artery evaluated by 64-slice multidetector CT (MDCT) of coronary arteries. To identify molecular biomarkers in the plasma, we performed a comprehensive plasma proteome profiling, liquid chromatography tandem mass spectrometry (LC-MS/MS), and targeted SRM/MRM.

\section{METHODS}

\section{Study participants and design}

The workflow of this study is summarized in figure 1 . Plasma samples from a discovery cohort $(\mathrm{n}=18)$, a verification cohort $(\mathrm{n}=53)$, and a validation cohort $(\mathrm{n}=228)$ were used here. The final-validation cohort included 218 asymptomatic subjects and 10 symptomatic subjects. The asymptomatic group included individuals who had undergone a coronary risk evaluation using 64-slide MDCT either for a health medical examination or for the evaluation of coronary artery disease concomitant with type 2 diabetes at the Seoul National University Bundang Hospital from March 2005 to May 2010. Moreover, we obtained plasma samples from 10 symptomatic patients who had undergone coronary artery bypass surgery because of typical chest pain associated with stenosis $\geq 50 \%$ in the three major coronary arteries. We identified their cardiovascular risk factors using baseline demographics and clinical profiles.

\section{Measurement of anthropometric and biochemical parameters} At the baseline, we measured body weight, height, and blood pressure (BP), and calculated body mass index (BMI) as the weight in kilogram divided by the square of the height in meter $\left(\mathrm{kg} / \mathrm{m}^{2}\right)$. Plasma samples were obtained from peripheral blood at the time of patient enrollment. All samples were stored at $-80^{\circ} \mathrm{C}$ until analysis. The plasma concentrations of total cholesterol, triglycerides, high-density lipoprotein cholesterol (HDLC), low-density lipoprotein cholesterol (LDL-C), aspartate aminotransferase, alanine aminotransferase, creatinine, glycated hemoglobin (HbAlc), high-sensitivity C reactive protein (hsCRP), and glucose were measured after overnight fast. The estimated glomerular filtration rate was calculated using the modification of diet and renal disease equation. ${ }^{6}$ We defined diabetes mellitus as follows: an $\mathrm{HbA1c}$ level $\geq 6.5 \%$, a fasting plasma glucose concentration of $\geq 126 \mathrm{mg} / \mathrm{dL}$ or taking any antidiabetic medication. Hypertension was defined as a systolic blood pressure of $>140 \mathrm{~mm} \mathrm{Hg}$, a diastolic blood pressure of $>90 \mathrm{~mm} \mathrm{Hg}$, or taking antihypertensive drugs. Dyslipidemia was defined as an LDL-C concentration of $\geq 130 \mathrm{mg}$ / $\mathrm{dL}$, a triglyceride concentration of $\geq 200 \mathrm{mg} / \mathrm{dL}$, or taking any lipid-lowering drugs.

\section{MDCT interpretation and definition of coronary artery stenosis} The 64-slice MDCT images were evaluated on a threedimensional image analysis workstation (Brilliance 64, Philips Medical Systems, Best, The Netherlands) according to the standard scanning protocol. Coronary artery stenosis was defined as a percentage of luminal stenosis of at least one major coronary artery. The severity of stenosis was defined in the following manner: normal coronary artery (no stenosis or stenosis in any of the coronary arteries $<10 \%$ ) (normal coronary artery stenosis group $(\mathrm{N})$ ), mild-to-moderate stenosis (mildto-moderate coronary stenosis group (M)) (stenosis in any of the coronary arteries between $11 \%$ and $50 \%$ ); and severe stenosis (severe coronary stenosis group (S)) (stenosis in all three coronary arteries $\geq 50 \%$ ). Patients who had undergone coronary artery bypass surgery were considered to be in the severe stenosis group.

\section{Experimental design and statistical rationale}

Discovery of candidate biomarkers of CAD using peptide labeling combined with tandem mass tag (TMT) and LC-MS/MS analysis

Proteins from plasma samples from the each of following six groups were pooled and immunodepleted using a multiple affinity removal system $\mathrm{Hu}-14$ column (Agilent Technologies, Santa Clara, California, USA), followed by in-solution protein digestion with trypsin (Promega, 

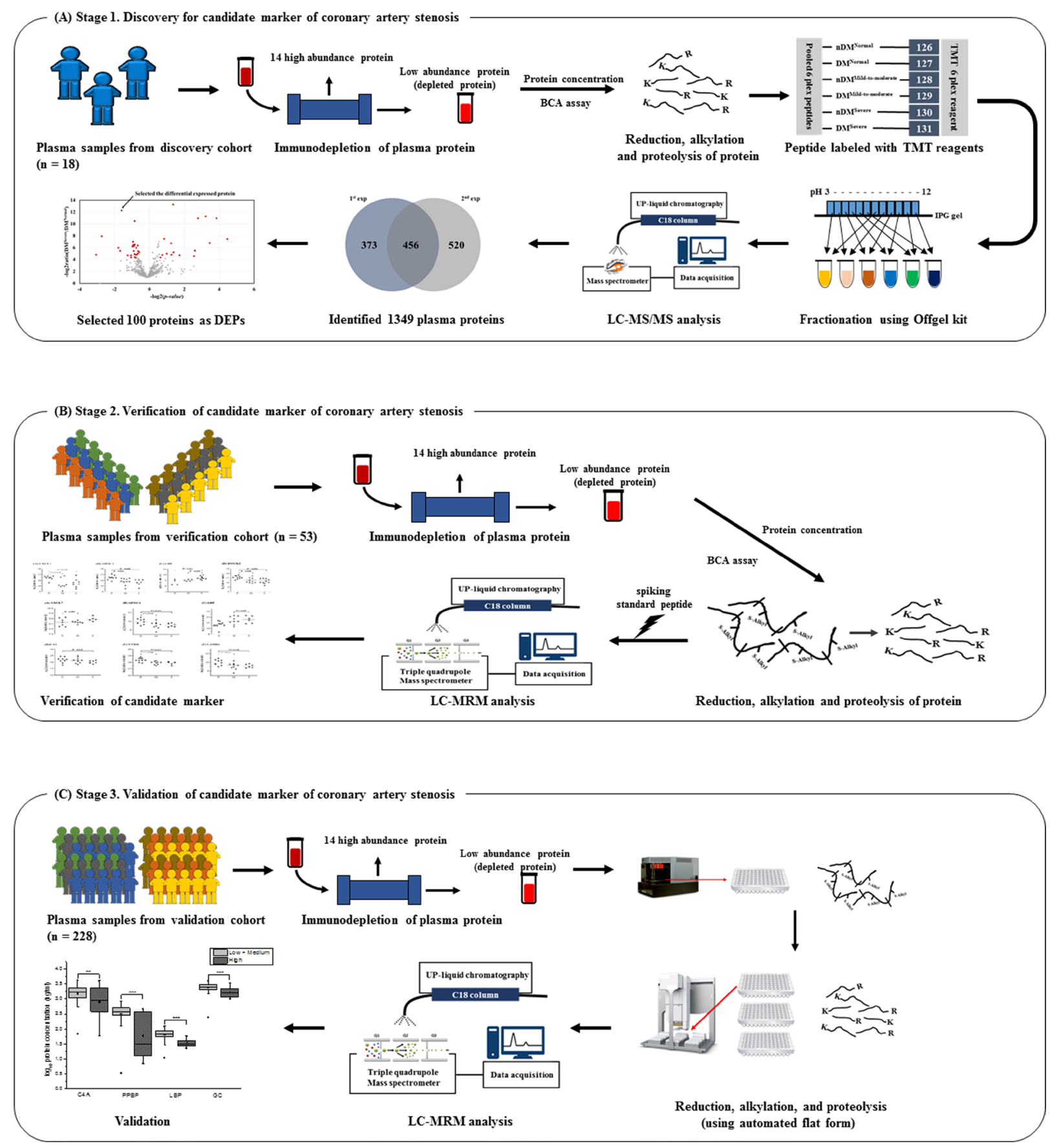

Figure 1 Workflow of the study. The figure shows the experimental strategy used for the discovery, verification, and validation of biomarkers of coronary artery stenosis. (A) Panel discovery stage. Plasma samples were collected from six groups that were classified according to the stage of coronary artery stenosis and presence/absence of diabetes mellitus as normal coronary artery $(N)$ without diabetes (non-DM) $(n=3)$, mild-to-moderate coronary stenosis $(M)$ with non-DM $(n=3)$, severe coronary artery stenosis $(S)$ with non-DM $(n=3)$, DM-N $(n=3), D M-M(n=3)$, and DM-S $(n=3)$. The pooled plasma samples from each group were labeled with TMT-6-plex reagents, respectively. An LC-MS/MS analysis was used to identify and semiquantify plasma proteomics. DEPs were selected based on statistical analyses. (B) Verification stage. Using DEPs, plasma samples that were previously depleted and digested for MRM analysis individually were selected from six groups: non-DM-N ( $n=10)$, non-DM-M $(n=10)$, non-DM-S $(n=3)$, DM-N $(n=10)$, DM-M $(n=10)$, and DM-S $(n=10)$. All samples were analyzed by label-free MRM, and 10 peptides were selected as candidate biomarkers. (C) Validation stage. Ten peptides were synthesized using isotope labeling by amino acids. The synthesized peptides were spiked in proteins prior to the digestion process. Plasma samples from the validation cohort $(n=228)$ were depleted individually. These processes, including reduction, alkylation, digestion, and desalting, were performed automatically. The concentration of peptides was calculated using spiking peptides. BCA, bicinchonic acid assay, DEP, differentially expressed protein; DM, diabetes mellitus; LC-MRM, liquid chromatography multiple reaction monitoring; LC-MS/MS, liquid chromatography tandem mass spectrometry; M, mild-to-moderate coronary stenosis group; MRM, multiple reaction monitoring; N, normal coronary artery stenosis group; S, severe coronary stenosis group; TMT, tandem mass tag; UPLC, ultra performacne liquid chromatography. 
San Jose, California, USA) peptide labeling with TMT 6-plex (Thermo Fisher Scientific, San Jose, California, USA) and off-gel fractionation (Agilent Technologies): (1) normal coronary artery without diabetes group (non-DM-N, n=3); (2) mild-to-moderate stenosis without diabetes group (non-DM-M, n=3); (3) severe stenosis without diabetes group (non-DM-S, n=3); (4) normal coronary artery with diabetes group (DM-N, n=3); (5) mild-to-moderate stenosis with diabetes group (DM-M, $\mathrm{n}=3$ ); and (6) severe stenosis with diabetes group (DM-S, $\mathrm{n}=3$ ) (figure 1). The peptides were analyzed using Q-exactive MS (Thermo Fisher Scientific) combined with Easy-nLC1000 (Thermo Fisher Scientific), as described previously. The raw data files were subjected to Proteome Discoverer V.1.4 (SEQUEST search algorithm) to assign the spectra matched to peptide against human proteome database (Uniprot/SwissProt; 20272 entries, released 22 January 2014). Peptide and protein probability were set as $95.0 \%$ and $99.9 \%$, respectively, with Scaffold to validate (protein false discovery rate $(\mathrm{FDR})<0.1 \%$ ). Identified proteins that matched at least two unique peptides were accepted. Intensities of TMT reporter ion were extracted and exported to spread sheet by Scaffold. Subsequently, the statistical analysis was performed using the R software package Isobar.

\section{Verification and validation of candidate markers}

All MRM experiments were performed on a 6490 triple quadrupole mass spectrometer (Agilent Technologies) coupled with a 1290 Infinity system (Agilent Technologies). The digested peptides were separated $(8 \mu \mathrm{g}$ on-column unless otherwise stated) by standard-flow reverse-phase ultra performance liquid chromatography (RP-UPLC) on a Zorbax Eclipse Plus C18 Rapid Resolution High Definition column $(150 \times 2.1 \mathrm{~mm}, 1.8 \mu \mathrm{m}$ particles; P/N. 959 759-902; Agilent Technologies) at $0.4 \mathrm{~mL} /$ min over a $43 \mathrm{~min}$ ACN gradient. The specific gradient was as follows (time, \%B): 0,$3 ; 1.5,7 ; 16,15 ; 18,15.3 ; 33$, $25 ; 38,45 ; 39,90 ; 42.9,90$; and 43, 3. The composition of the mobile phases were $0.1 \%$ formic acid (FA) for A and $0.1 \%$ F.A in ACN for B. The spray voltage was set to $2.5 \mathrm{kV}$, and the temperature of nitrogen drying gas was set to $250^{\circ} \mathrm{C}$. The column and autosampler, which were contained within a 1290 Infinity system (Agilent Technologies), were maintained at $50^{\circ} \mathrm{C}$ and $4^{\circ} \mathrm{C}$, respectively.

We performed the label-free MRM analysis to verify the candidate marker for coronary artery stenosis using the verification cohort. A total of 53 plasma samples from 10 patients per groups with the exception of non-DM-S group ( $n=3$ ) were subject to MRM analyses following immunodepletion, in-solution digestion and spiking of standard peptide (LFTGHPETL*EK). Prepared samples were analyzed using label-free MRM analysis individually, and then peak areas of peptides were normalized to that of the standard peptide.

In the protein digestion step, we adopted an automateddigestion system to remove the technical variation and to increase reproducibility. Plasma samples were depleted of high abundant proteins and $40 \mu \mathrm{g}$ of the protein were used for the study. The samples were processed for proteolytic digestion and desalting using an automated platform (Bravo Automated Liquid Handling Platform, Agilent) capable of handling 96 samples in parallel based on filter-aided sample preparation (FASP) method. The protocol has been split into four parts Alkylation, washing, proteolysis and peptide elution. Briefly, the protein samples were reduced with tris (2-caboxyethyl) phosphine (TCEP) for $30 \mathrm{~min}$ at $37^{\circ} \mathrm{C}$ in an Eppendorf and transferred to the 96-well polyethersulfone (PES) membrane plates for automatic processing (Pall Life Sciences, AnnArbor, Michigan, USA). The reduced proteins were subjected to vacuum (10 in $\mathrm{Hg}$ for $15 \mathrm{~min}$ ) to remove the buffer, and alkylated with indole-3-acetic acid (IAA) for $40 \mathrm{~min}$ at room temperature (RT). The buffer was eluted with vacuum (10 in $\mathrm{Hg}$ for $15 \mathrm{~min}$ ) and was washed with $8 \mathrm{M}$ urea and $50 \mathrm{mM}$ ammonium bicarbonate $(\mathrm{ABC})$ solution three times and vacuum pressure was performed to attain buffer exchanges at $10 \mathrm{in} \mathrm{Hg}$ for $15 \mathrm{~min}$. Proteolysis was effected by adding trypsin at 1:50 trypsin to protein ratio. Two pmol of SI peptides were spiked into the samples after addition of trypsin. The eluted peptides were desalted using C-18 cartridges for 96 samples with the automated platform as reported by us previously with a different cartridge. The eluted peptides were dried using a SpeedVac and stored in $-20^{\circ} \mathrm{C}$ until analysis.

In the validation experiment, we used eight stable isotope (SI)-labeled synthetic peptides (Thermo Fisher Scientific, ${ }^{13} \mathrm{C}^{15} \mathrm{~N}$-lysine or arginine; purity, $\left.>97.0 \%\right)$. The transition of SI peptides was optimized using Skyline (MacCoss lab software 3.0), and the elution time of SI peptides on a column was determined. The SI peptides with eight points of varying concentrations by making serial dilutions of highest point using diluted matrix to achieve the following final concentrations: $0.003,0.01$, $0.03,0.1,0.3,1.0,3.0$ and 10.0 pmol.

\section{Statistical analyses}

Statistical analyses were performed using the SPSS V.20.0 software for Windows and the GraphPad Prism V.4.0 package (La Jolla, California, USA). Categorical variables are presented as frequencies and percentages, and continuous variables are expressed as means \pm SD. After performing a Kolmogorov-Smirnov normality test, significant differences between the diabetes and non-diabetes groups were analysed using a two-sided, unpaired Student's t-test for normally distributed variables or the Mann-Whitney U test for non-normally distributed variables. The data acquired using MS were analyzed using the Proteome Discoverer software with the SEQUEST search engine and Isobar ( $\mathrm{R}$ package) were used. All raw data of peak areas from MRM analyses were extracted using the Skyline tool. All of the measured variables were analyzed separately for each group according to the grade of coronary artery stenosis. In a verification cohort, t-test was used to verify the candidate proteins in 
Table 1 Clinical characteristics of the subjects for the validation cohort stratified by the severity of coronary artery stenosis $(n=228)$

\begin{tabular}{|c|c|c|c|c|c|c|}
\hline Variables & $\begin{array}{l}\text { Normal } \\
(n=99)\end{array}$ & $\begin{array}{l}\text { Mild-to-moderate } \\
\text { stenosis }(n=95)\end{array}$ & $\begin{array}{l}\text { Severe stenosis } \\
(n=34)\end{array}$ & $P$ value & $\begin{array}{l}\text { Post hoc } \\
\text { analysis }\end{array}$ & $\begin{array}{l}\mathrm{P} \text { for } \\
\text { trend }\end{array}$ \\
\hline Age (years) & $53.4 \pm 8.2$ & $59.1 \pm 8.8$ & $63.3 \pm 10.0$ & $<0.001$ & $a, b, c$ & \\
\hline Male (\%) & $43(49.4)$ & $80(77.7)$ & $24(82.8)$ & $<0.001$ & & $<0.001$ \\
\hline BMI $\left(\mathrm{kg} / \mathrm{m}^{2}\right)$ & $24.7 \pm 3.0$ & $25.0 \pm 2.8$ & $24.1 \pm 2.7$ & 0.417 & & \\
\hline Systolic blood pressure $(\mathrm{mm} \mathrm{Hg})$ & $123.7 \pm 15.4$ & $125.8 \pm 10.1$ & $124.4 \pm 18.3$ & 0.882 & & \\
\hline \multicolumn{7}{|l|}{ Comorbidities } \\
\hline Diabetes mellitus (\%) & $24(27.6)$ & 31 (30.1) & $10(34.5)$ & 0.769 & & \\
\hline Hypertension (\%) & $17(19.5)$ & $27(26.2)$ & $13(44.8)$ & 0.027 & & 0.041 \\
\hline Dyslipidemia (\%) & $12(13.8)$ & $16(15.5)$ & $9(31.0)$ & 0.088 & & 0.621 \\
\hline \multicolumn{7}{|l|}{ Laboratory values } \\
\hline HbA1c (\%) & $6.3 \pm 1.4$ & $6.4 \pm 1.5$ & $6.8 \pm 1.9$ & 0.336 & & \\
\hline Total cholesterol (mg/dL) & $210.2 \pm 44.8$ & $206.8 \pm 39.5$ & $185.2 \pm 40.4$ & 0.017 & b, c & \\
\hline Triglyceride (mg/dL) & $170.2 \pm 242.7$ & $167.9 \pm 118.2$ & $167.0 \pm 57.7$ & 0.950 & & \\
\hline HDL-C (mg/dL) & $53.7 \pm 11.9$ & $50.2 \pm 12.7$ & $45.8 \pm 9.2$ & 0.003 & $\mathrm{~b}$ & \\
\hline LDL-C (mg/dL) & $111.8 \pm 29.6$ & $113.8 \pm 33.3$ & $109.6 \pm 22.2$ & 0.890 & & \\
\hline AST (IU/L) & $26.2 \pm 8.9$ & $24.6 \pm 8.0$ & $24.7 \pm 10.5$ & 0.488 & & \\
\hline ALT (IU/L) & $30.6 \pm 18.5$ & $31.4 \pm 20.1$ & $21.5 \pm 9.2$ & 0.012 & $b, c$ & \\
\hline eGFR $\left(\mathrm{mL} / \mathrm{min} / 1.73 \mathrm{~m}^{2}\right)$ & $80.8 \pm 15.4$ & $74.4 \pm 15.2$ & $81.6 \pm 27.2$ & 0.077 & & \\
\hline Albumin-to-creatinine ratio $(\mathrm{g} / \mathrm{mg})$ & $11.3 \pm 18.7$ & $132.0 \pm 14.39$ & $111.34 \pm 442.02$ & 0.562 & & \\
\hline hsCRP (mg/dL) & $0.17 \pm 0.34$ & $0.32 \pm 1.15$ & $2.30 \pm 5.05$ & $<0.001$ & $b, c$ & \\
\hline
\end{tabular}

Data are presented as the mean \pm SD. hsCRP was analysed by log transformation.

ALT, alanine aminotransferase; AST, aspartate aminotransferase; BMI, body mass index; eGFR, estimated glomerular filtration rate; HDL-C, high-density lipoprotein cholesterol; hsCRP, high-sensitivity C reactive protein; LDL-C, low-density lipoprotein cholesterol.

between groups. To examine independently the effect of the proteins or peptides that predict $\mathrm{CAD}$, an analysis of the covariance was performed in the final validation cohort. In each model, age, sex, BMI, LDL-C, and hsCRP were adjusted as covariates. In this study, significance was set at $\mathrm{p}<0.05$.

\section{RESULTS}

\section{Baseline characterisation of the cohorts}

The age, BMI, systolic BP, proportion of sex difference, comorbidities, and biochemical profile of the subjects for the final validation cohort were categorized according to the degree of coronary artery stenosis and are presented in table 1 .

A total of 228 samples were analysed in this study; 99 subjects with normal coronary artery, 95 subjects with mild-to-moderate coronary stenosis, and 34 subjects with severe stenosis. The mean age was higher in subjects with severe stenosis than it was in subjects with normal and mild-to-moderate coronary stenosis $(63.3 \pm 10.0$ years for severe stenosis; $59.1 \pm 8.8$ years for mild-tomoderate stenosis; and $53.4 \pm 8.2$ years for normal coronary arteries). The proportion of males and the ratio of previous hypertension increased with increasing severity of stenosis. As expected, the severe stenosis group exhibited a higher hsCRP and a lower HDL-C compared with the normal and mild-to-moderate stenosis groups. The presence of dyslipidemia, plasma level of LDL-C, and urinary albumin-to-creatinine ratio were not significantly different among the groups. The previous discovery cohort $(n=18)$ and the verification cohort $(n=53)$ showed baseline characteristics that were similar to those of the final validation cohort (see online supplementary tables S1 and S2).

\section{Identification and selection of signature peptides as} candidate biomarkers of coronary artery stenosis via plasma protein profiling

We pooled the individual plasma samples from three subjects from each group and labeled them with TMT6 plex reagents as follows: non-DM-N group with 126, nonDM-M group with 128, non-DM-S group with 130, DM-N group with 127, DM-M group with 129, and DM-S group with 131. A total of 1349 different proteins were identified from the pooled samples (see online supplementary table S3). Based on the results of the TMT quantitative analysis, we selected differentially expressed proteins (DEPs) with a $\mathrm{p}<0.05$ among the identified proteins and a change ratio $\geq 1.5$ fold in at least one replicate from duplicate LC-MS/MS analysis. Fifty-two proteins were selected as candidate markers of coronary artery stenosis in patients without diabetes (see online supplementary 
table S4), and 69 proteins were selected in patients with diabetes (see online supplementary table S5).

\section{Quantification of candidate biomarkers via label-free MRM analysis for verification of DEPS}

A plasma protein quantification analysis was performed to select biomarkers according to the grade of coronary artery stenosis and the presence of type 2 diabetes mellitus. Among DEPs identified from the discovery cohort, we selected the signature peptides representing candidate protein biomarkers which have sufficient signal sensitivity, suitability to assess in MRM analysis. To normalize and compare peak areas obtained from different mass runs, we added $2.5 \mathrm{pmol}$ of isotopically labeled standard peptide, LFTGHPETL*EK. Total of 108 transitions derived from 36 peptides representing 27 proteins were selected from the tested 414 transitions from 125 peptides representing 100 proteins including DEPs and known marker proteins when satisfying the following criteria: (1) three transitions per peptide were coeluted; (2) dot-product score above 0.8; (3) signal to noise $(\mathrm{S} / \mathrm{N})$ value above 10 in triplicate MRM run (see online supplementary table S6 and figure S1).

After t-test analyses between groups, four proteins including chemokine (C-X-C motif) ligand 7 (CXCL7), apolipoprotein C-II (APOC2), human lipopolysaccharidebinding protein (LBP), and dedicator of cytokinesis 2 (DOCK2), were selected as candidate biomarker proteins for the vascular stenosis in patients with diabetes with $\mathrm{p}<0.05$ (figure 2). Six proteins, including CXCL7, APOC2, LBP, complement component 4A (C4A), vitamin D-binding protein (VTDB), and laminin $\beta 1$ subunit (LAMB1), were selected as candidate biomarker proteins for the vascular stenosis in patients without diabetes (figure 3).

\section{Validation of the selected biomarkers of coronary artery} stenosis via MRM analysis with isotopically labeled peptides For validation of seven candidate biomarker proteins for diagnosis of vascular stenosis in patients with or without

diabetes, we performed the labeled MRM analysis with plasma samples from an independent set of 228 patients based on the criteria that were used during the discovery phase. For the labeled MRM assay, seven SI peptides, which represent the seven candidate proteins, were used. The transition and elution time of SI were optimized using MRM assay, and we get the concentration curve of SI peptides using MRM assay (see online supplementary table $\mathrm{S} 7$ and figure S2).

After immunodepletion, the $500 \mathrm{fmol}$ of seven SI peptides were added to each sample before digestion. The MRM analysis was performed three technical replicates for each sample and sequence of sample run was made up randomly. Based on peak area ratios (transition $^{\text {endogenous peptide }} /$ transition $^{\text {SI peptide }}$ ), we calculated the concentration of endogenous peptides/proteins.

Furthermore, the changes in concentration of candidate proteins were analyzed according to the degree of stenosis and the presence/absence of diabetes. After a full adjustment for conventional risk factors of $\mathrm{CAD}$, we identified four upregulated proteins (APOC2, CXCL7, DOCK2, and VTDB) and one downregulated protein $(\mathrm{C} 4 \mathrm{~A})$ in the diabetes group and three downregulated proteins (CXCL7, LBP, and VTDB) in the non-diabetes group (table 2).

In subjects with diabetes, APOC2 tended to increase as increase of degree of stenosis (fold change: 6.55 in the DM-S group vs DM-N group, $\mathrm{p}=0.005 ; 2.09$ in the DM-S group vs DM-M group, $\mathrm{p}=0.084$; and 3.13 in the DM-M group vs DM-N- group, $\mathrm{p}=0.024)$. CXCL7 showed upregulation in the DM-S group (fold change: 2.81 in the DM-S group vs the DM-N group, $\mathrm{p}=0.013$; and 2.62 in the DM-S group vs the DM-M group, $\mathrm{p}=0.009$ ). DOCK2 was upregulated by twofold in the severe stenosis group compared with the normal coronary artery group and mild-tomoderate stenosis group (fold change: 2.43 in the DM-S group vs the DM-N group, $\mathrm{p}=0.009$; and 2.00 in the DM-S group vs the DM-M group, $\mathrm{p}=0.016)$. VTDB was upregulated in the mild-to-moderate stenosis group compared

(C) LBP

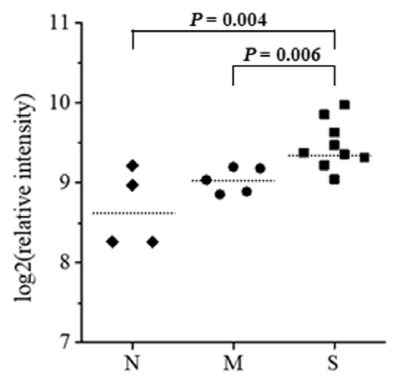

(D) DOCK2

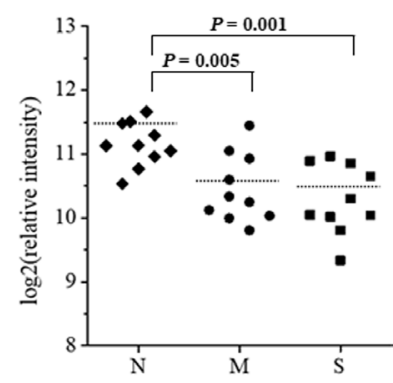

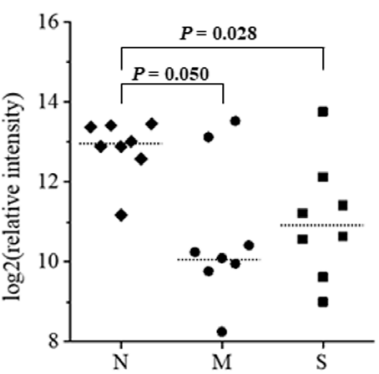

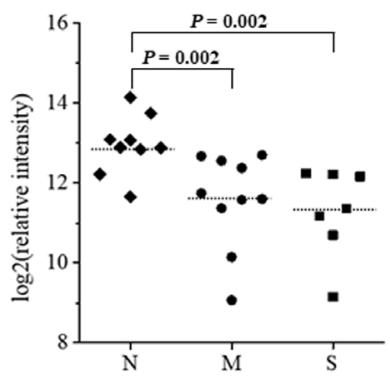

Figure 2 Selection of candidate biomarkers of coronary artery stenosis in subjects with diabetes using a label-free MRM analysis. The scatter plots show the differential expression of genes between the normal, mild-to-moderate coronary stenosis, and severe coronary stenosis groups. CXCL7, APOC2, LBP, and DOCK2 were selected as candidate biomarkers of coronary artery stenosis in patients with diabetes. t-Test was used to verify the candidate proteins in between groups. ${ }^{*} \mathrm{P}<0.05$. APOC2, apolipoprotein C-II; CXCL7, chemokine (C-X-C motif) ligand 7; DOCK2, dedicator of cytokinesis 2; LBP, lipopolysaccharidebinding protein; M, mild-to-moderate coronary stenosis group; MRM, multiple reaction monitoring; N, normal coronary artery stenosis group; S, severe coronary stenosis group. 
(A) $\mathrm{CXCL}_{7}$

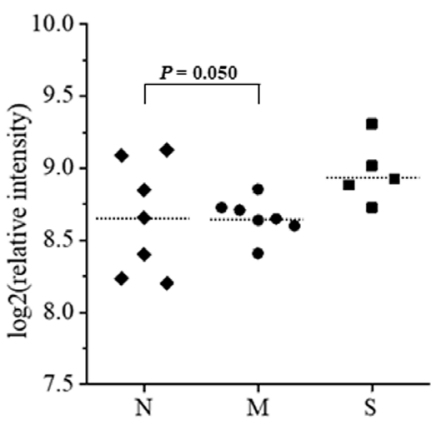

(D)C4A

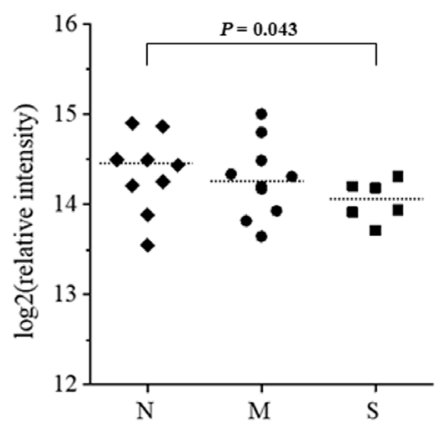

(B) $\mathrm{APOC} 2$

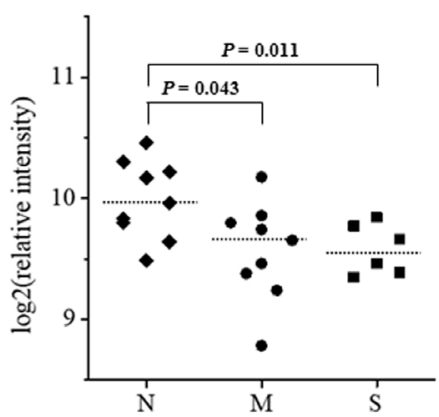

(E) VTDB

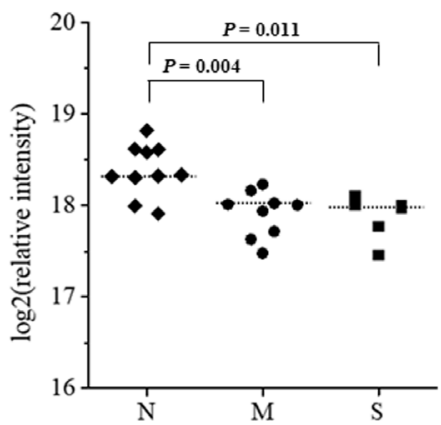

(C) LBP

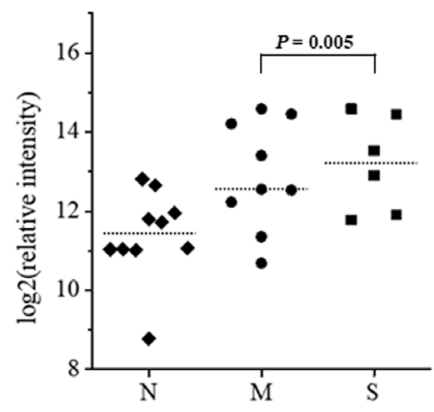

(F) LAMB1

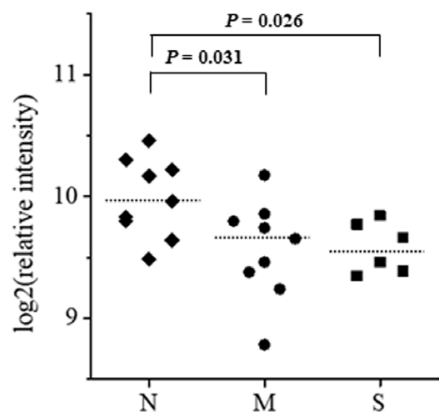

Figure 3 Selection of candidate biomarkers of coronary artery stenosis in subjects without diabetes using a label-free MRM analysis. The figure shows the differential expression of genes between the normal, mild-to-moderate coronary stenosis, and severe coronary stenosis groups. CXCL7, APOC2, LBP, C4A, VTDB, and LAMB1 were selected as candidate biomarkers of coronary artery stenosis in subjects without diabetes. t-test was used to verify the candidate proteins in between groups. ${ }^{*} \mathrm{P}<0.05$. APOC2, apolipoprotein C-II; C4A, complement component 4A; CXCL7, chemokine (C-X-C motif) ligand 7; LAMB1, laminin $\beta 1$ subunit; LBP, lipopolysaccharide-binding protein; $M$, mild-to-moderate coronary stenosis group; MRM, multiple reaction monitoring; $\mathrm{N}$, normal coronary artery stenosis group; $\mathrm{S}$, severe coronary stenosis group; VTDB, vitamin D-binding protein.

with the normal coronary group (fold change: 1.34 in the DM-M group vs the DM-N group, $\mathrm{p}<0.001)$. C4A was downregulated by 3 to 4 -fold in the severe stenosis group compared with the normal or mild-to-moderate stenosis group (fold change: 3.37 in the DM-S group vs the DM-N group, $\mathrm{p}=0.003$; and 3.81 in the DM-S group vs the DM-M group, $\mathrm{p}<0.001)$.

In subjects without diabetes, LBP and VTDB were downregulated in the severe stenosis group compared with the normal and mild-to-moderate stenosis group (fold change: 1.47 in the non-DM-S vs the non-DM-N, $\mathrm{p}=0.005 ; 1.63$ in the non-DM-S vs the non-DM-M, $\mathrm{p}<0.001$, for LBP; 1.21 in the non-DM-S vs the non-DM-N, $p=0.036$; 1.29 in the non-DM-S vs non-DM-N, $\mathrm{p}=0.009$, for VTDB, respectively). In the mild-to-moderate stenosis group of non-diabetic subjects, CXCL7 showed downregulation (fold change: 1.53 in the non-DM-S vs the non-DM-M, $\mathrm{p}=0.012$ ).

\section{DISCUSSION}

In the present study, we identified significant associations between upregulated plasma APOC2, CXCL7, DOCK2 and VTDB levels and the downregulated C4A level and the development of CAD in subjects with diabetes. Also, we found the relationship between downregulated plasma CXCL7, LBP and VTDB levels and the severity of CAD in subjects without diabetes, as assessed by a proteomics analysis using the MRM technique. MS is an emerging technique in the field of proteomics investigations because of its excellent selectivity and sensitivity. Although CVD is the leading cause of death in patients with diabetes, few studies have identified early-detectable and predictable biomarkers. To our knowledge, this is the first study that used an updated proteomics analysis of variation in the levels of plasma proteins according to the severity of coronary artery stenosis, followed by a stringent validation of the selected candidate biomarkers in a different set of patients with and without diabetes.

As results of this study, meaningful biomarkers could be refined with early and late markers depending on the severity of coronary artery stenosis. In diabetic patients, the increase of APOC2 and VTDB were significant in the comparison with the normal coronary artery group and mild-to-moderate coronary stenosis group, and there was no statistically significant marker in the non-diabetic 

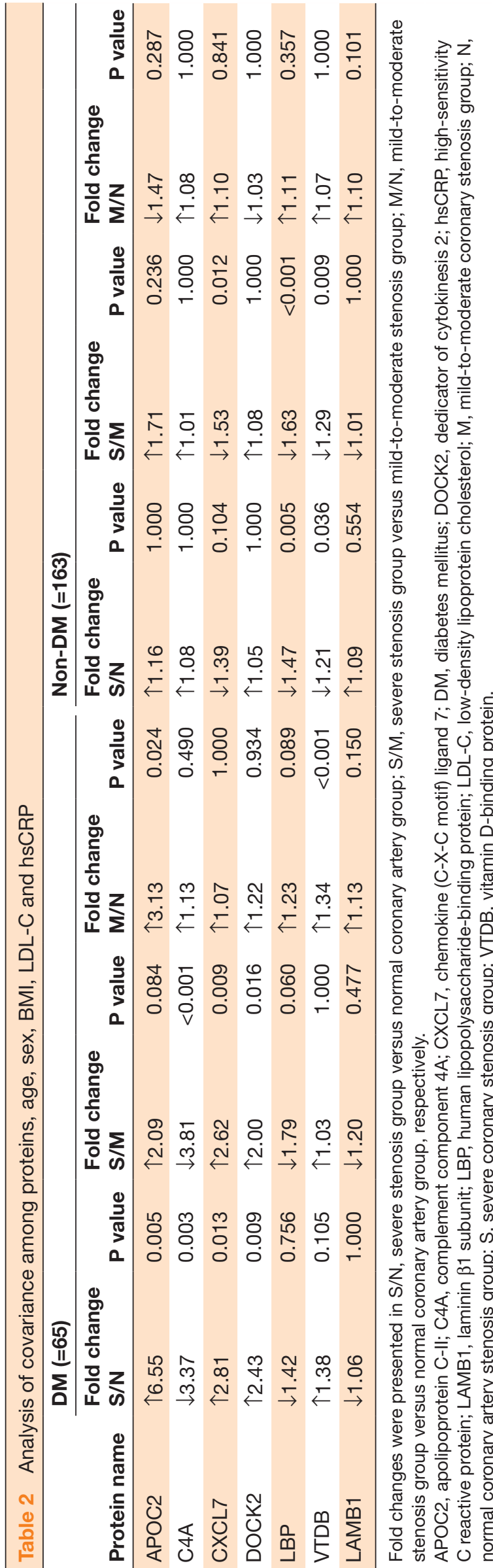

patients. We noted the upregulated APOC2 and VTDB in diabetes as subclinical atherosclerosis markers.

APOC2 is an activator of lipoprotein lipase (LPL) in triglyceride metabolism. ${ }^{7}$ A rare autosomal recessive mutation in APOC2 causes hypertriglyceridemia because of the alteration in hydrolysis and removal of triglycerides from chylomicrons and very-low-density lipoproteins. ${ }^{7}$ Our findings suggest that upregulation of APOC2 is an independent predictive risk factor for CAD in diabetes. This is in contrast with a previous relationship observed between the levels of APOC2 and CAD. Notably, because APOC2 was upregulated in the mild-to-moderate stenosis group compared with the normal coronary artery group in this study, it may represent an early detectable biomarker of coronary artery stenosis. Although we did not examine the translation of APOC2 in specific tissues, this result may explain the compensatory mechanism for regulating increased triglyceride-rich lipoprotein particles in insulin-resistant patients.

Interestingly, VTDB showed an opposite tendency according to the presence of diabetes. In diabetic subjects, VTDB was upregulated in the mild-to-moderate coronary stenosis group compared with the normal coronary group, indicating the possibility of early biomarker for CAD as well as APOC2. Epidemiological studies have suggested the existence of an inverse association between the levels of 25-hydroxyvitamin $\mathrm{D}$ and the risk of CAD in humans. ${ }^{8}$ However, limited data are available on the role of VTDB in CAD. A recent meta-analysis showed that polymorphisms of codon 416 and codon 420 of VTDB were associated with susceptibility to type 2 diabetes in Asians but not in Caucasians. ${ }^{10}$ However, in another study, single nucleotide polymorphism of VTDB did not show a significant association with $\mathrm{CAD}$, unlike low 25-hydroxyvitimin D. ${ }^{11}$ According to the African American Diabetes Heart study, VTDB measured by ELISA did not show a significant association with subclinical CVD represented by calcified plaque of coronary arteries and carotid arteries. ${ }^{12}$ Regarding to the results reported so far, the role of VTDB on CVD is controversial. The present study suggested that upregulated VTDB could be a biomarker for predicting subclinical atherosclerosis in diabetes, through the MRM proteomics, which is highly sensitive and specific. According to the present results, a low level of VTDB was an independent predictive marker of severe CAD in individuals without diabetes. VTDB is the main transporter of vitamin $\mathrm{D}$, and approximately $90 \%$ of the circulating 25-hydroxyvitamin $\mathrm{D}$ is bound to VTDB, for transfer into target cells. ${ }^{13}$ Thus, VTDB may be a reservoir for vitamin D deficiency, as shown in studies using VTDB knockout mice. ${ }^{14}$ In addition to transportation, VTDB plays an important role in the elimination of damaged cells and the activation of immune cells in the inflammatory status, as well as in the transport of vitamin D. ${ }^{15}$ As stated previously, inflammation is supposed to be a significant contributor to atherosclerosis; thus, we suggest that a low level of circulating VTDB is a predictor of CAD in patients without diabetes. 
On the other hand, significant biomarkers in the severe stenosis group are carefully proposed as reflecting the severity of CAD: downregulated C4A and upregulated DOCK2 and CXCL7 for the subjects with diabetes; and downregulated LBP and VTDB for the subjects without diabetes.

Increasing evidence suggests a significant role for systemic low-grade chronic inflammation in the pathogenesis of CAD ${ }^{16}$ However, little is known about the association between C4A deficiency and the development of $\mathrm{CAD}$. A previous study reported that the classical complement pathway, in particular the C4 protein, was deregulated in degenerative diseases. ${ }^{17}$ We have also reported previously that the serum concentration of the complement component C1q was significantly lower in patients with severe coronary artery stenosis compared with the normal control group. ${ }^{18}$ Clq plays a major role in triggering activation by enzymatic cleavage of $\mathrm{C} 4$ to $\mathrm{C} 4 \mathrm{a}$ and C4b; thus, it is linked to C4A levels. ${ }^{19}$ In this study, we observed a significant downregulation of $\mathrm{C} 4 \mathrm{~A}$ after adjustment for cardiometabolic risk factors, including the well-known inflammatory biomarker hsCRP. This reflected the fact that deficiency of C4A altered the immune reaction linked to $\mathrm{CAD}$ independently, which was supported by another study that reported that $\mathrm{C} 4 \mathrm{~A}$ deficiency decreases the clearance of immune complexes and triggers serious complications after cardiopulmonary bypass surgery in pediatric patients. ${ }^{20}$ We suggest the downregulation of C4A as a novel biomarker of future $\mathrm{CAD}$ in patients with diabetes.

Recent studies have indicated that DOCK2 regulates cellular processes associated with cell adhesion, proliferation, apoptosis, and, especially, the migration and activation of lymphocytes, neutrophil chemotaxis, and the development of innate immune cells. ${ }^{21}$ We found that DOCK2 levels were mildly elevated in individuals with mild-to-moderate stenosis and observed a significant trend toward the upregulation of this protein in the severe stenosis group. To date, few studies have addressed the association between DOCK2 and CAD. Recently, Guo et al reported that DOCK2 expression was activated in injury-induced smooth muscle cells and involved vascular remodeling. ${ }^{22}$ Our findings support the notion that the elevation of DOCK2 levels may be a predictable severe marker of the development of $\mathrm{CAD}$ in diabetes.

In the present study, CXCL7 exhibited a trend toward upregulation according to the severity of coronary artery stenosis in diabetes, after adjustment for strong risk factors for CAD. Several studies have suggested that CXCLs and CXC receptors exhibit a relationship with cardiomyopathy, hypertension, and atherogenesis. ${ }^{23}{ }^{24} \mathrm{~A}$ recent study reported that the activated CXC chemokine receptor 3 and its ligands participated in atherosclerosis and adverse cardiac remodeling. ${ }^{23}$ Several studies have suggested that the proinflammatory chemokines CXCL2, CXCL3, CXCL5, and CXCL10 are positively associated with metabolic syndrome, including non-alcoholic fatty liver disease. ${ }^{25-27}$ However, among non-diabetic subjects, the moderate stenosis group alone showed a higher level of CXCL7; thus, future studies of its clinical implications in humans are needed.

The last biomarker to be mentioned is the downregulated LBP in the non-diabetic subjects. LBP is an acute-phase reactant, binds lipopolysaccharide, so has been considered a surrogate biomarker for the lipopolysaccharide-induced innate immunity activation. Some studies reported that plasma LBP level was associated with $\mathrm{CAD}$, type 2 diabetes and peripheral arterial stiffness. ${ }^{28}{ }^{29}$ The present study showed downregulated LBP was associated with severe coronary artery stenosis in the non-diabetic group. Recently, Pretorius et al presented that the formation of fibrin and amyloid in the clot was reversed after addition of LBP to the clot structures affected by lipopolysaccharide in type 2 diabetes. ${ }^{30}$ It is noteworthy that the recent study showing the reversibility of LBP to atherogenesis to account for the downregulation of LBP in subjects with severe coronary artery stenosis observed in our results.

\section{Strengths and limitations}

MRM (which is sometimes called SRM) is a mass spectroscopy method that is emerging as the approach of choice for targeting selective peptides for the detection and quantification of a protein. Compared with conventional ELISA, MRM assays have many advantages for the verification and validation of large numbers of biomarkers and the simultaneous direct measurement of panels of candidate biomarkers of disease. The MRM method for biomarker discovery plays a powerful biological role in the identification of candidate markers of pathophysiology and targets of drugs to treat diseases.

In the validation cohort, we made our best to exclude the effect of statin treatment on our discovered secretory proteins. The majority of validation cohort consisted of drug-naive patients or of whom had health examination. However, three patients from coronary artery bypass graft $(\mathrm{CABG})$ surgery with statin therapy were included. Even though we cannot completely remove the statin influence, it was minimized as possible as we could. The present study was limited by the sample size, the nature of the proteomics analysis, and restricted ethnicity. Large-scale studies of individuals from various ethnic groups are needed to confirm our results. Despite this limitation, the present study suggested the meaning of candidate proteins as focused in the pathophysiology and their clinical implications, especially according to the degree of coronary artery stenosis with risk stratification. Furthermore, MRM analyses using serum circulating proteins and peptides are highly sensitive and specific, thus allowing the development of a biomarker panel kit for the early detection of CAD. In addition, our candidate proteins were stringently validated in a different set of patients (more than 200 subjects). The practical use of our technique in developing a multi-ELISA kit for the early diagnosis of CAD is expected. 


\section{CONCLUSION}

In conclusion, our MRM proteomics analysis identified five related proteins that are potential early diagnostic biomarkers of coronary artery stenosis. The upregulation of APOC2, CXCL7, DOCK2 and VTDB and the downregulation of $\mathrm{C} 4 \mathrm{~A}$ are significant biomarkers for CVD in patients with diabetes. The downregulation of LBP and VTDB are suggested as predictive biomarkers for CAD in non-diabetic individuals. Notably, we suggest upregulated APOC2 and VTDB as potential biomarkers of subclinical coronary atherosclerosis in diabetes.

\section{Author affiliations \\ ${ }^{1}$ Internal Medicine, Chungbuk National University Hospital, Cheongju, South Korea ${ }^{2}$ Internal Medicine, Chungbuk National University College of Medicine, Cheongju,} South Korea

${ }^{3}$ Applied Chemisty, Kyung Hee University College of Applied Sciences, Yongin, South Korea

${ }^{4}$ Thoracic and Cardiovascular Surgery, Seoul National University Bundang Hospital, Seongnam, South Korea

${ }^{5}$ Thoracic and Cardiovascular Surgery, Seoul National University College of Medicine, Seoul, South Korea

${ }^{6}$ Pharmaceutics, Gachon University College of Pharmacy, Incheon, South Korea ${ }^{7}$ Urology, Seoul National University Bundang Hospital, Seongnam, South Korea ${ }^{8}$ Urology, Seoul National University College of Medicine, Seoul, South Korea ${ }^{9}$ Internal Medicine, Seoul National University Bundang Hospital, Seongnam, South Korea

${ }^{10}$ Internal Medicine, Seoul National University College of Medicine, Seoul, South Korea

Contributors SHC and KPK conceived and designed the experiments. EJK and $\mathrm{K}-\mathrm{CC}$ acquired and analyzed the data, and wrote the manuscript. K-CC, JWK, JWO, YRC, J-MP, and N-YH performed the experiments. CL, JJO, TJO, HCJ, HL and SHC contributed materials/analysis tools. All authors contributed to completion of the manuscript. All authors read and approved the final manuscript.

Funding This work was supported by the Korea Health Technology R\&D Project through the Korea Health Industry Development Institute (KHIDI), funded by the Ministry of Health and Welfare, Republic of Korea (grant number Hl11V0005-020013); the Medical Research Center through the National Research Foundation of Korea funded by the Ministry of Science (grant number NRF2018R1A5A2024425); ICT and future Planning, and Kyung Hee University Medical Center (grant number HI15C-1595-020017); the Korea Science and Engineering grant funded by Korea government (grant number 201824425); and VHS Medical Center Research Grant, Republic of Korea (grant number: VHSMC 19032).

\section{Competing interests None declared.}

Patient consent for publication Not required.

Ethics approval This study was conducted in accordance with the Declaration of Helsinki and was approved by the ethics committees of SNUBH (SNUBH IRB \#B-1203/147-006, \#A111218CP02). All subjects provided their written informed consent.

Provenance and peer review Not commissioned; externally peer reviewed.

Data availability statement All data relevant to the study are included in the article or uploaded as supplementary information.

Open access This is an open access article distributed in accordance with the Creative Commons Attribution Non Commercial (CC BY-NC 4.0) license, which permits others to distribute, remix, adapt, build upon this work non-commercially, and license their derivative works on different terms, provided the original work is properly cited, appropriate credit is given, any changes made indicated, and the use is non-commercial. See: http://creativecommons.org/licenses/by-nc/4.0/.

\section{ORCID iDs}

Eu Jeong Ku http://orcid.org/0000-0001-5533-4989

Hak Chul Jang http://orcid.org/0000-0002-4188-6536

Sung Hee Choi http://orcid.org/0000-0003-0740-8116
REFERENCES

1 World health Organization G. Part II global health indicators: 1. life expectancy and mortality. World health Statics. Available: http://www.who.int/gho/publications/world_health_statistics/EN WHS2015_Part2.pdf [Accessed 20 Dec 2019]

2 Centers for Disease Control and prevention. National diabetes statistics report, 2017. Available: https://www.cdc.gov/diabetes/ $\mathrm{pdfs} /$ data/statistics/national-diabetes-statistics-report.pdf [Accessed 20 Dec 2019].

3 Jude EB, Oyibo SO, Chalmers N, et al. Peripheral arterial disease in diabetic and nondiabetic patients: a comparison of severity and outcome. Diabetes Care 2001;24:1433-7.

4 Paterson RW, Heywood WE, Heslegrave AJ, et al. A targeted proteomic multiplex CSF assay identifies increased malate dehydrogenase and other neurodegenerative biomarkers in individuals with Alzheimer's disease pathology. Transl Psychiatry 2016;6:e952.

5 Picotti P, Aebersold R. Selected reaction monitoring-based proteomics: workflows, potential, pitfalls and future directions. Nat Methods 2012;9:555-66.

6 Fuiano G, Zoccali C, Bertoni E, et al. [Guidelines for ambulatory monitoring of kidney transplant patients. Adaptation of the Guidelines of the American Society of Transplantation (J Am Soc Nephrol 2000; 11 (S1): 86)]. G Ital Nefrol 2004;21:S11-50.

7 Cox DW, Breckenridge WC, Little JA. Inheritance of apolipoprotein C-Il deficiency with hypertriglyceridemia and pancreatitis. N Engl J Med 1978;299:1421-4

8 Hoge A, Donneau A-F, Streel S, et al. Vitamin D deficiency is common among adults in Wallonia (Belgium, $51^{\circ} 30^{\prime}$ North): findings from the nutrition, environment and cardio-vascular health study. Nutr Res 2015;35:716-25.

9 Gurses KM, Tokgozoglu L, Yalcin MU, et al. Markers of subclinical atherosclerosis in premenopausal women with vitamin D deficiency and effect of vitamin D replacement. Atherosclerosis 2014;237:784-9.

10 Wang G, Li Y, Li L, et al. Association of the vitamin D binding protein polymorphisms with the risk of type 2 diabetes mellitus: a metaanalysis. BMJ Open 2014;4:e005617.

11 Michos ED, Misialek JR, Selvin E, et al. 25-Hydroxyvitamin D levels, vitamin $D$ binding protein gene polymorphisms and incident coronary heart disease among whites and blacks: the ARIC study. Atherosclerosis 2015;241:12-17.

12 Freedman BI, Divers J, Russell GB, et al. Vitamin D associations with renal, bone, and cardiovascular phenotypes: African American-Diabetes heart study. J Clin Endocrinol Metab 2015;100:3693-701.

13 White P, Cooke N. The multifunctional properties and characteristics of vitamin D-binding protein. Trends Endocrinol Metab 2000;11:320-7.

14 Christakos S, Ajibade DV, Dhawan P, et al. Vitamin D: metabolism. Endocrinol Metab Clin North Am 2010;39:243-53.

15 Delanghe JR, Speeckaert R, Speeckaert MM. Behind the scenes of vitamin D binding protein: more than vitamin D binding. Best Pract Res Clin Endocrinol Metab 2015;29:773-86.

16 Luc G, Bard J-M, Juhan-Vague I, et al. C-reactive protein, interleukin-6, and fibrinogen as predictors of coronary heart disease: the prime study. Arterioscler Thromb Vasc Biol 2003;23:1255-61.

17 Xu Y, Yan J, Zhou P, et al. Neurotransmitter receptors and cognitive dysfunction in Alzheimer's disease and Parkinson's disease. Prog Neurobiol 2012;97:1-13.

18 Hong ES, Lim C, Choi HY, et al. The amount of C1q-adiponectin complex is higher in the serum and the complex localizes to perivascular areas of fat tissues and the intimal-medial layer of blood vessels of coronary artery disease patients. Cardiovasc Diabetol 2015;14:50.

19 Schumaker VN, Zavodszky P, Poon PH. Activation of the first component of complement. Annu Rev Immunol 1987;5:21-42.

20 Zhang S, Wang S, Li Q, et al. Capillary leak syndrome in children with C4A-deficiency undergoing cardiac surgery with cardiopulmonary bypass: a double-blind, randomised controlled study. Lancet 2005;366:556-62.

21 Love PE, Bhandoola A. Signal integration and crosstalk during thymocyte migration and emigration. Nat Rev Immunol 2011:11:469-77.

22 Guo X, Shi N, Cui X-B, et al. Dedicator of cytokinesis 2, a novel regulator for smooth muscle phenotypic modulation and vascular remodeling. Circ Res 2015;116:e71-80.

23 Altara R, Manca M, Brandão RD, et al. Emerging importance of chemokine receptor CXCR3 and its ligands in cardiovascular diseases. Clin Sci 2016;130:463-78. 
24 Borst O, Schaub M, Walker B, et al. CXCL16 is a novel diagnostic marker and predictor of mortality in inflammatory cardiomyopathy and heart failure. Int J Cardiol 2014;176:896-903.

25 Park JH, Lee DH, Park MS, et al. C-C chemokine receptor type 5 deficiency exacerbates alcoholic fatty liver disease through pro-inflammatory cytokines and chemokines-induced hepatic inflammation. J Gastroenterol Hepatol 2017;32:1258-64.

26 Xu Z, Zhang X, Lau J, et al. C-X-C motif chemokine 10 in nonalcoholic steatohepatitis: role as a pro-inflammatory factor and clinical implication. Expert Rev Mol Med 2016;18:e16.
27 Zhang X, Han J, Man K, et al. CXC chemokine receptor 3 promotes steatohepatitis in mice through mediating inflammatory cytokines, macrophages and autophagy. J Hepatol 2016;64:160-70.

28 Sakura T, Morioka T, Shioi A, et al. Lipopolysaccharide-binding protein is associated with arterial stiffness in patients with type 2 diabetes: a cross-sectional study. Cardiovasc Diabetol 2017;16:62.

29 Lepper PM, Schumann C, Triantafilou K, et al. Association of lipopolysaccharide-binding protein and coronary artery disease in men. J Am Coll Cardiol 2007;50:25-31.

30 Pretorius E, Mbotwe S, Kell DB. Lipopolysaccharide-Binding protein (LBP) reverses the amyloid state of fibrin seen in plasma of type 2 diabetics with cardiovascular co-morbidities. Sci Rep 2017;7:9680. 